Check for updates

Cite this: RSC Adv., 2017, 7, 41429

Received 12th July 2017

Accepted 19th August 2017

DOI: 10.1039/c7ra07650a

rsc.li/rsc-advances

\section{Bio nano ink for 4D printing membrane proteins $\uparrow$}

\author{
Anu Stella Mathews, (D) ab Sinoj Abraham, (D) ab Surjith Kumar Kumaran, ${ }^{\text {ab Jiaxin Fan }}{ }^{\text {bc }}$ \\ and Carlo Montemagno*ab
}

The transfer of bio-functionality from natural living organisms to stable engineered environments opens a wide horizon of applications. Our work focusses on the creation of materials and devices that transform bio traits and collect, process and act on the information in response to changes in their local environment, thus promoting additive manufacturing from 3D space to a four-dimensional, functional space. In this work we have expanded the set of tools enabling the incorporation of biological function as an intrinsic property in the devices we print with a new class of light curable bio nano ink. This novel approach allows the 3D printing of acrylic polymer compositions containing biological materials, especially, membrane proteins using a photo $(407 \mathrm{~nm})$ curing stereolithography (SLA)/digital light processing (DLP) 3D printer. Retaining the bio functionality of these proteins gives a fourth dimensional (4D) aspect to this construct. Herein, we report the 4D printing of a bio-inspired nano hybrid electrode for water-splitting applications using a polymeric resin with proton-pumping bacteriorhodopsin (bR), silver nanoparticles (Ag NP) and carbon nanotubes (CNT). These printed photo electrochemical cells exhibit high durability, low onset over potential, and upon light irradiation $(535 \mathrm{~nm}$ ) produces hydrogen by a synergistic effect of Ag NP and bR.

\section{Introduction}

The additive process of distributing successive material layers in 3D shapes has advanced the revolutionary effects of the rapid dissemination of text, images, and information from 2D printing, there-by transforming science and education. The 3D printing process allows the mass customization of lighter, stronger, and complex structures in a sustainable, environmentally friendly, energy efficient manner. ${ }^{1}$ The recent advances in material science, cell biology and engineering have helped the development of stable biological building blocks in 3D spaces, thus enabling them to disseminate biological functions and portray the fourth dimension. The emerging pathways in four dimensional (4D) printing ${ }^{2-16}$ either mimics the dynamic architecture of nature by incorporating stimuli responsive materials such as shape memory alloys ${ }^{5-8}$ and swellable hydrogel composites ${ }^{9-11}$ for self-folding origami ${ }^{12-14}$ or focuses on smart scaffolds for regenerative medical applications. ${ }^{15-17}$ Apart from the existing $4 \mathrm{D}$ printing techniques, we introduce a new direction in the fourth dimension, i.e. function. The 3D printed

\footnotetext{
angenuity Lab, 11421 Saskatchewan Drive, Edmonton, Alberta T6G 3M9, Canada. E-mail: montemag@ualberta.ca; Tel: +1-780-641-1617

${ }^{b}$ Department of Chemical and Materials Engineering, University of Alberta, Edmonton, Alberta, T6G 2V4, Canada

'Department of Electrical and Computer Engineering, University of Alberta, Edmonton, Alberta, T6G 2V4, Canada
}

$\dagger$ Electronic supplementary information (ESI) available. See DOI: 10.1039/c7ra07650a structures using bioinks process the ability to retain the inherent property of the biomolecule in the structure and mimic its function as in the biological system. Among the existing printing methods, like extrusion-based, robotic dispensing, cellular inkjet, laser-assisted printing and integrated tissue organ printing, ${ }^{18}$ stereolithographic printing has the advantage of the solid freeform fabrication technique attaining precise geometry of submicron-sized structures and decimeter-sized objects as designed using computer aided interfaces and/or medical imaging data forms. ${ }^{19,20}$ Our work focuses on the next evolution that capitalizes on the advancement of 4D stereolithographic printing to enable the creation of materials and devices that transform energy, process and act on the information in response to changes in their local environment. Designed ink is a bio-nano hybrid with optimized compositions of acrylic polymer resin, silver nanoparticles (Ag $\mathrm{NP}$ ), carbon nanotubes (CNT) and photo initiator, implanting the fourth dimension of function as a photo electrochemical cell for water-splitting by transferring the intrinsic property of the membrane protein bacteriorhodopsin (bR) to the printed structure.

The quest for renewable, sustainable and non-polluting energy sources starts with the photo electrochemical splitting of water by titanium dioxide, ${ }^{21}$ which found an analogy with natural photosynthesis, thanks to nature's photon-driven proton pump bacteriorhodopsin (bR). ${ }^{22-24}$ The direct electrochemical hydrogen evolution reactions of electrodes fabricated by in situ electro-less deposition of Ag NPs on top of the solid carbon cloth supported bR monolayers provides promising 
features for design of novel hybrid catalysts with improved activity and durability. ${ }^{25}$ In present work, we exploit, for the first time, advanced three-dimensional (3D) printing to create a bioinspired hybrid nanocomposite electrode for water splitting application. The stability of bacteriorhodopsin, in detergent free milieu, is ensured by wrapping with amphiphilic beta-sheet peptide with an acrylic moiety, therefore covalently crosslinking the protein in the printed polymeric hydrogel. The additive manufacturing of this novel hybrid electrode uses UV curable bio-nano ink was successfully conducted and wielded for direct bio photo-electrochemical (BPEC) water splitting. The printed materials possess the shape of three dimensional structures placed together with the bio-functionality of photo electrochemical cells within optimized device flexibility, paving a new perspective for 4D printed bio-material apart from shape memory or tissue compatibility. The current study also offers the potential expansion for a various proteins and for making artificial materials that can reproduce biological functionality.

\section{Results and discussion}

\section{Bio-nano ink}

For all three central approaches for $4 \mathrm{D}$ printing (i.e. biomimicry, autonomous self-assembly and tissue engineering) to succeed, material research including the careful design of ink and the optimization of composition is the fundamental block to be laid. Scheme 1 shows the bio-nano ink development involved in this work.

2-Hydroxyethyl methacrylate (2-HEMA), well known as the first monomer to synthesize hydrogels for biomedical applications with di(ethylene glycol)dimethacrylate (dEGdMA) is used as the ink base. Di(trimethylolpropane)tetraacrylate (dTMP4A), a tetra functional monomer, is employed as cross linker providing a fast cure response and a high cross link density upon UV curing. $1: 1$ ratio of 2-HEMA : dEGdMA and varying composition of dTMP4A (0-30\%) is employed as the acrylic base due to the versatility the polymer can offer with regards to its structural stability, water absorption and biological activity. Diphenyl(2,4,6-trimethylbenzoyl)phosphine oxide/2-hydroxy-2-

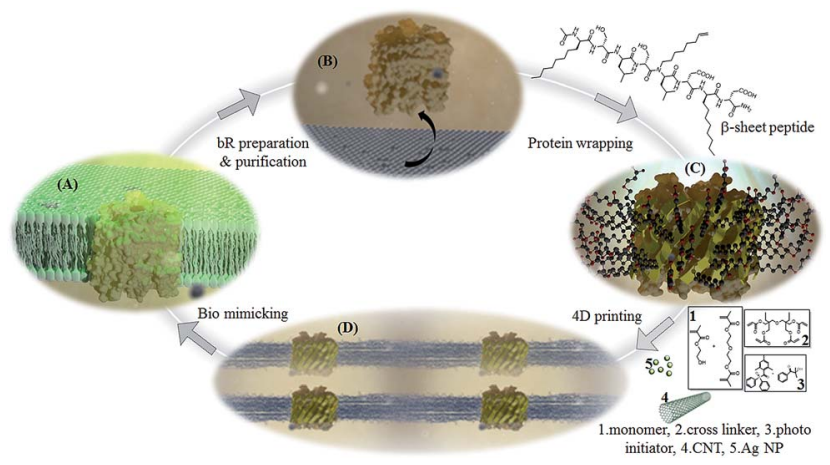

Scheme 1 Bio-nano ink preparation (A) natural proton pump, membrane containing bacteriorhodopsin (B) bR protein expressed out of its natural membrane (C) stabilization of bR by wrapping with vinyl $\beta$-sheet peptide (D) bio-nano ink composition containing monomers, cross linker, photo initiator, bR, CNT and Ag NP, 3D printed at $405 \mathrm{~nm}$. methylpropiophenone-blend is the initiator utilized to initiate the UV curing through free radical mechanism. The $\beta$-sheet peptide wrapped bacteriorhodopsin $(\beta \mathrm{P}-\mathrm{bR})$ functions as the bio aspect whereas the well reported boost in photo conversion efficiency imparted by single walled carbon nanotube ${ }^{30}$ and the enhanced hydrogen evolution reaction under irradiation at $550 \mathrm{~nm}$ due to the synergic effect of $\mathrm{Ag} \mathrm{NP}$ and $\mathrm{bR}^{25}$ makes CNT and $\mathrm{Ag}$ NP the nano facet of the 4D printed bio photo electrochemical cell (BPEC). The major challenge to achieve the fourth dimension is the incorporation of bR into the ink by improvising its stability and bio-function to a detergent free, vesicle/ micelle devoid hydrogel phase. Since short amphiphilic $\beta$-strand peptides are reported to stabilize integral membrane proteins, ${ }^{26}$ we have designed the sequence, acetyl-(octyl) $\operatorname{GSLSL}(N$-1-octene)D(octyl)GD-NH , with the traits of which are first, alternating hydrophilic and hydrophobic faces of 8 amino acid residues that are optimal to span the central non polar membrane bilayer region, ${ }^{27}$ second octyl elongated side chains to implicate lipo "peptitergent" design, ${ }^{28}$ third the $N$-1-octene substitution of leucine in the 5 th position to have the duel merit of enhanced inter-strand association by fibrillogenesis inhibition $^{29}$ and forth with acryl functionalization of the stabilized bR for UV curing. The optimal 4D printing composition of the bionano ink was found to acrylic base (93.0 wt\%), photo-initiator (1 wt\%), $\beta$ P-bR ( $1 \mathrm{wt} \%)$, Ag NP (2 wt $\%$ ) and CNT (3 wt $\%)$. The optimal acrylic base composition contains equal portions of 2-HEMA and dEGdMA and 15\% of crosslinker dTMP4A.

\section{Experimental characterizations}

The discussion of the material characterizations of Ag NP, vinyl$\beta$-sheet peptide, and $\beta \mathrm{P}-\mathrm{bR}$ are in detail in the ESI section. $\dagger$ Fig. 1 shows the elemental mapping, surface morphology conducted using scanning electron microscopy-energy dispersive Xray spectrometry (SEM-EDS) and Fourier transform infrared spectroscopy (FTIR) studies of the 3D printed BPEC. Hydrogel at a lower resolution of $1 \mathrm{~mm}$ is homogeneous in nature (Fig. 1A), whereas at a higher resolution of $2.00 \mu \mathrm{m}$ has nanoclusters (Fig. 1B). The Energy Dispersive X-ray (EDX) mapping reveals
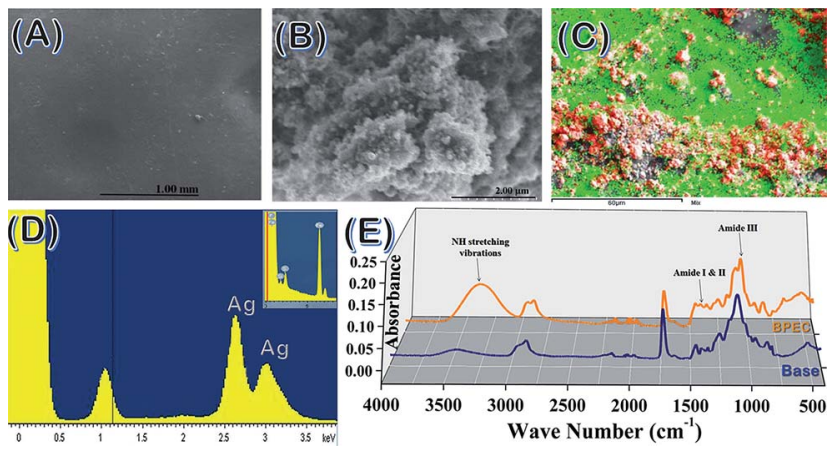

Fig. 1 Surface morphology and elemental mapping of 3D printed BPEC. (A and B) Surface morphology at $1.00 \mathrm{~mm}$ and $2.00 \mu \mathrm{m}$ resolution (C) elemental mapping of surface at $60 \mu \mathrm{m}$ (D) EDX analysis showing the presence of silver nano particles (insert shows baseline) and (E) FTIR spectra of 3D printed discs showing presence of protein. 
that there are peaks of silver around $3 \mathrm{keV}$, which confirm Ag NP exists in the composition (Fig. 1C). FTIR spectra, Fig. 1D, of 3D printed BPEC shows clearly the incorporation of protein evident from the NH stretching vibrations, amide I, II and III peaks in comparison with base (containing acrylic base with $15 \%$ cross linker).

Differential scanning calorimetric (DSC) and thermo gravimetric (TGA) traces of the 3D printed bio-nano ink discs were taken with/without overnight soaking in PBS buffer ( $\mathrm{pH}$ 7.4) to determine the thermal properties. The weight loss of $20 \%$ $(T 20 \%)$ for the samples before overnight soaking (BPEC) changed at $403{ }^{\circ} \mathrm{C}$ followed by $94 \%$ weight loss up to $472{ }^{\circ} \mathrm{C}$ whereas the disc after soaking in water $(\mathrm{BPEC}(\mathrm{W}))$ showed a rapid weight loss of $17 \%$ from $80-170{ }^{\circ} \mathrm{C}$, which can be attributed to the water trapped inside the hydrogel, trailed by another $T(20 \%)$ from 170 to $402{ }^{\circ} \mathrm{C}, 76 \%$ weight loss until $449{ }^{\circ} \mathrm{C}$. The final decomposition temperatures of both BPEC and BPEC(W) are in the range $445-475^{\circ} \mathrm{C}$ as plotted in Fig. 2A. The DSC curves of BPEC and BPEC $(\mathrm{W})$ from temperature $-50^{\circ} \mathrm{C}$ to $300{ }^{\circ} \mathrm{C}$ are presented in Fig. 2B. Generally, DSC analysis shows similarity in thermal behavior of all prepared discs. However, some differences for presoaked disc can be observed characterized by endothermic peak with maximum in the range 83.3-125.4 ${ }^{\circ} \mathrm{C}$ which are connected with the loss of water present in the hydrogel structure. Rheology measurements showed changes in the storage modulus $\left(G^{\prime}\right)$ and loss modulus $\left(G^{\prime \prime}\right)$ as a function of thickness of the BPEC printed, Fig. 2C and D. $G^{\prime}$ measures the deformation energy stored during shear process of a test material which is characteristic of the stiffness of the material and $G^{\prime \prime}$ is representative of the energy dissipated during shear which is characteristic of the flow response of the
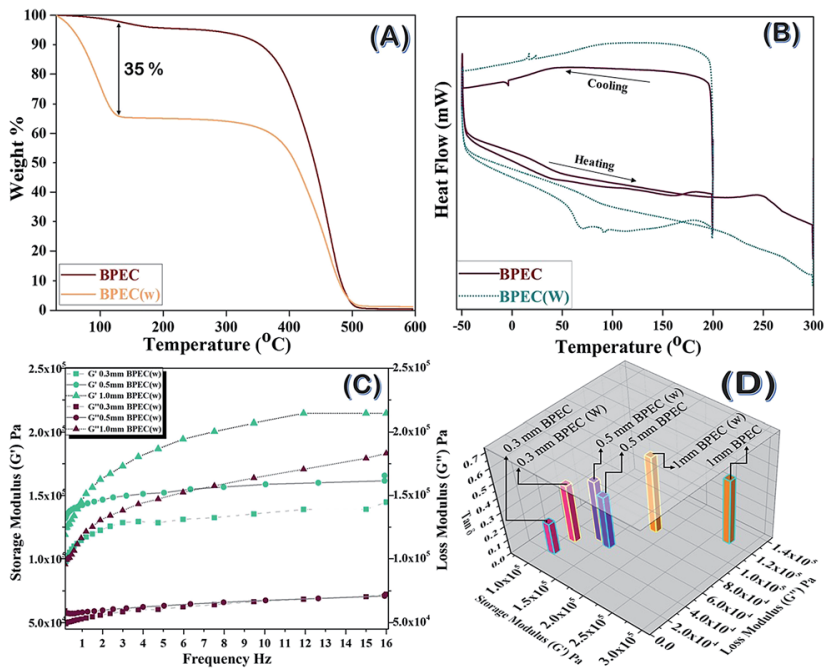

Fig. 2 Thermal and rheological properties of 3D printed bio-nano ink discs BPEC, without water and BPEC(W), after overnight soaking in buffer (A) TGA curves of $5 \mathrm{~mm}$ diameter, $0.5 \mathrm{~mm}$ thick $7-8 \mathrm{mg}$ discs, (B) DSC graphs of $3 \mathrm{~mm}$ diameter, $0.3 \mathrm{~mm}$ thick, $4-5 \mathrm{mg}$ discs (C) storage modulus $\left(G^{\prime}\right)$ and loss modulus $\left(G^{\prime \prime}\right)$ versus frequency plot of $25 \mathrm{~mm}$ diameter $\times 1 \mathrm{~mm}, 0.5 \mathrm{~mm}$ and $0.3 \mathrm{~mm}$ discs after overnight water soaking (D) variation in $G^{\prime}, G^{\prime \prime}$ and $\tan \delta$ of the discs before and after water soaking. material. The $G^{\prime}$ of the discs increases at elevated thickness which can be attributed to the extended crosslinking resulting in diminished macromolecular network flexibility and expanded material rigidity due to the direct interdependence of storage modulus with rigidity. $G^{\prime}$, irrespective of disc thickness, decreased after soaking, implying the flexibility brought to the macromolecular layers by the incorporation of water moieties. $G^{\prime}$ was much higher than that of $G^{\prime \prime}$ due to the formation of elastic hydrogel from crosslinking. The ratio, $G^{\prime \prime} / G^{\prime}=\tan \delta$ is less than $1\left(G^{\prime}>G^{\prime \prime}\right)$, indicating the printed material to be elastic solid, whereas $\tan \delta$ values increases after overnight buffer soaking showing enhancement of viscous liquid behavior due to water molecules trapped inside the hydrogel.

\section{Four-dimensional additive manufacturing}

Stereolithographic printing using a $405 \mathrm{~nm}$ LED source engine is used to print different structures for the present work as shown in Scheme 2.

The 3D modelling of the STereoLithography (STL) files is done to attain the desired designs and is printed using the bionano inks. The printing resolution determination was done by varying the cross linker and the curing time for spike and hole models with a gradient diameter of 1-0.1 mm and length/depth of $1 \mathrm{~mm}$, which were analyzed using field emission scanning electron microscopy (FE-SEM) (Fig. 3). Surface studies of the printed samples show that the best resolution obtained was successful $1 \mathrm{~mm}$ printing of $0.2 \mathrm{~mm}$ spikes and $0.2 \mathrm{~mm}$ through holes into the design with $15 \%$ of cross linker and $3 \mathrm{~s}$ of layer by layer curing as optimum. A lower concentration of cross linker as well as curing time between layers failed to deliver a solid framework to the printed architecture while above $20 \%$ concentration of cross linker in bio-nano ink and $>3 \mathrm{~s}$ cure time resulted in extensively cured holes and brittle spikes sacrificing the desired flexibility and design of the architecture.

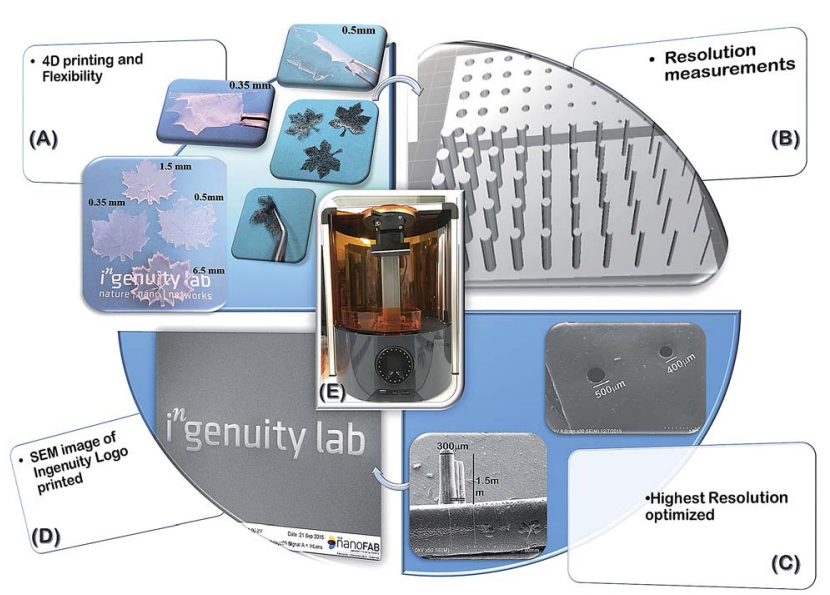

Scheme 2 Different structures printed using the 3D printer for various analysis (A) maple leaf with different thickness using base ink (transparent) and bio-nano ink (black) (B) STL file of the spike and hole model printed for resolution analysis, (C) SEM images showing the smallest holes and spikes printed (D) SEM image of Ingenuity lab emblem printed and (E) image of stereolithographic printer used. 

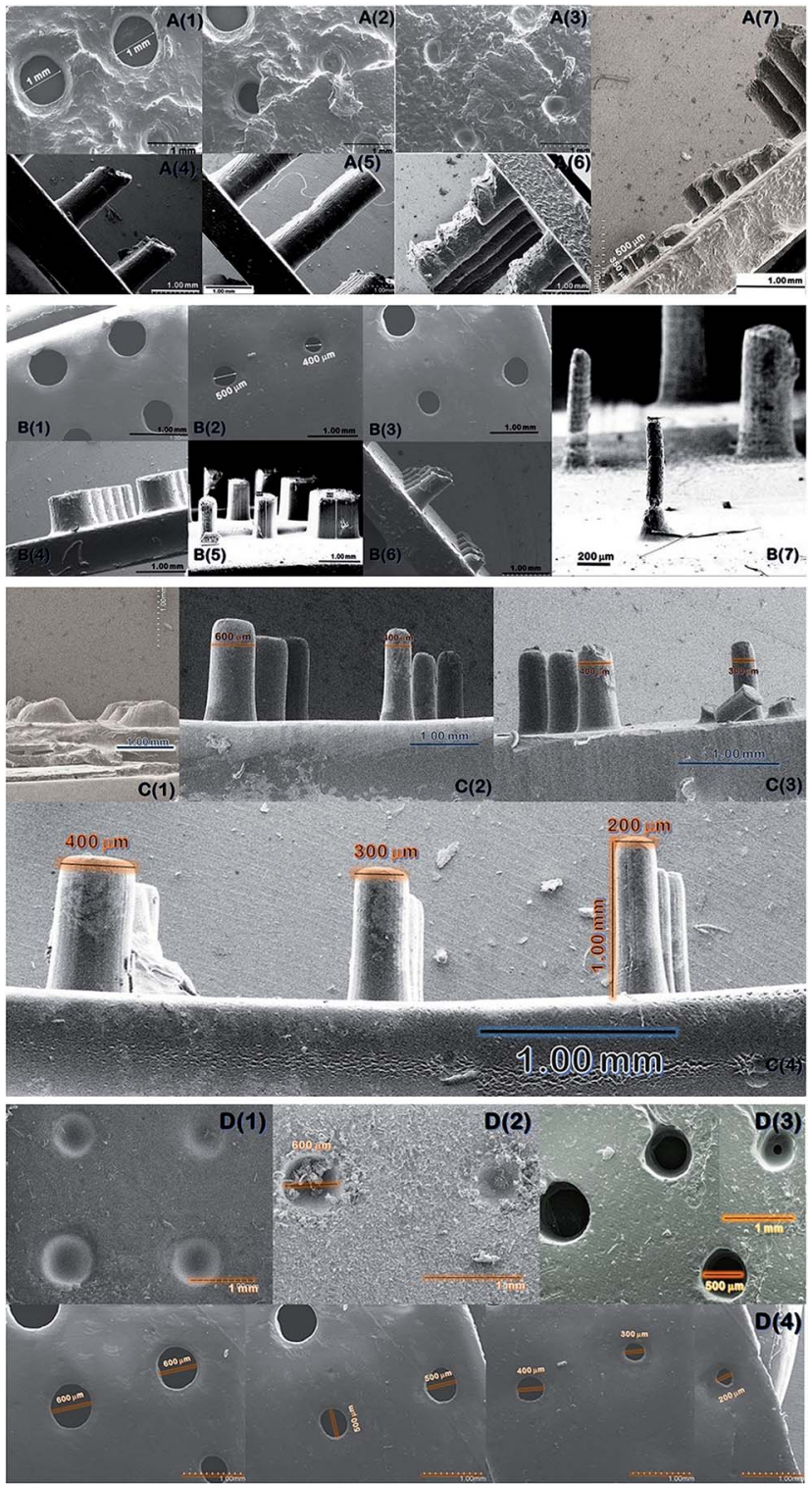

Fig. 3 FE-SEM images of 3D printed spikes and holes for cross-linker amount and cure rate related resolution (A) $30 \%$ cross linker containing base ink printed with various model exposure cure time (1) $2.8 \mathrm{~s}$ (2) $3.0 \mathrm{~s}$, (3) $4.0 \mathrm{~s}$, (4) $2.5 \mathrm{~s}$ (5) $2.8 \mathrm{~s}$ (6) $4 \mathrm{~s}$ (7) $3 \mathrm{~s}$ and (B) $20 \%$ cross linker containing base ink printed with various model exposure cure time (1) $2.8 \mathrm{~s}$ (2) $3.0 \mathrm{~s}$, (3) $4.0 \mathrm{~s}$, (4) $2.5 \mathrm{~s}$ (5) $2.8 \mathrm{~s}$ (6) $4 \mathrm{~s}$ (7) $3 \mathrm{~s}$ (C) spikes printed with $3 \mathrm{~s}$ model exposure cure time using base ink containing various cross linker amount (1) $0 \%$ (2) $5 \%$ (3) $10 \%$ (4) $15 \%$ (D) holes printed with $3 \mathrm{~s}$ model exposure cure time using base ink containing various cross linker amount (1) $0 \%$ (2) 5\% (3) $10 \%$ (4) $15 \%$.

\section{Bio photo electrochemical cell}

$4 \mathrm{D}$ printed structures were tested for its reproducibility of bio function and are tested for water splitting efficiency and hydrogen evolution quantification in the bio photoelectrochemical cell (BPEC) illustrated in Fig. 4. The hydrogen generation measurements are carried out at room temperature $\left(25 \pm 2{ }^{\circ} \mathrm{C}\right)$ in $1.0 \mathrm{M}$ PBS buffer at $\mathrm{pH}$ 7.4. The designs with thicker layers failed to generate any hydrogen while the constructs with $0.3-0.5 \mathrm{~mm}$ thickness is successfully employed
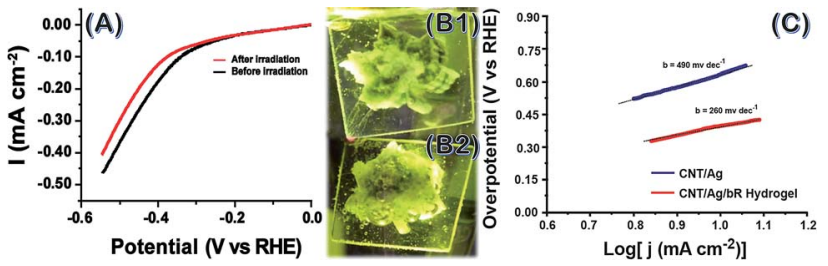

Fig. 4 Water splitting studies of BPEC. (A) Electrochemical activity of hydrogel in the form of polymerization curve before and after $535 \mathrm{~nm}$ light irradiation in $1 \mathrm{M}$ PBS solution at $\mathrm{pH} 7.4$, (B) BPEC in shape of maple leaf generating bubbles indication $\mathrm{H}_{2}$ generation (B1) before and (B2) after protein incorporation and (C) Tafel plot of the hydrogel electrodes with (-) and without (-) bR protein incorporation.

as BPEC with the preference of $0.5 \mathrm{~mm}$ ones printed on an ITOcoated glass electrode. The polarization curve before and after $535 \mathrm{~nm}$ light irradiation on the hydrogel electrode (Fig. 4A) was showed that the overpotential of the hydrogel electrode is $84 \mathrm{mV}$ at a current density of $1 \mathrm{~mA} \mathrm{~cm} \mathrm{~cm}^{-2}$ after irradiating with light. The electrocatalytic analyses in the form of Tafel plots are depicted in Fig. 4C, in which the linear region fitted into Tafel equation (detailed experiments and analysis were described in $\mathrm{ESI} \dagger$ ). The hydrogel contains AgNP/bR/CNT electrode exhibit Tafel slope $\sim 260 \mathrm{mv} \mathrm{dec}^{-1}$ in the region $327-432 \mathrm{mV}$. The Tafel slope, often been used to identify the hydrogen evaluation reaction mechanism and its rate-determining step measured in this work closely matches that reported on hydrogels prepared in carbon cloth suggesting a hydrogen adsorption in the surface is rate-determining step. The formation of hydrogen generation was observed under $535 \mathrm{~nm}$ light irradiation on the 3D printed hydrogel. Fig. 4C depicts the accumulative amount of $\mathrm{H}_{2}$ produced $v s$. time in presence and absence of protein. During 30 min light irradiation, in the presence of $\mathrm{bR}, 100 \mathrm{~mL}$ of $\mathrm{H}_{2}$ was obtained in comparison with $3 \mathrm{~mL}$ of $\mathrm{H}_{2}$ produced without protein. This confirms that the synergic combination of $\mathrm{bR}, \mathrm{Ag}$ NP and CNT's are important for efficient water splitting and more over the functionality of the bio-moiety is intact in the $3 \mathrm{D}$ printed bio-nano ink composition contributing the fourth dimension to the printed structure (here maple leaf). Previously, it has been shown that the incorporation of silver nanoparticles on to beta sheet peptide wrapped bR protein provides the system dynamic and possesses a higher energy value, in which the switching of intermediate photo cycle of bR in hundreds of nanoseconds compared to millisecond for pristine form. ${ }^{25,31}$ The presence of silver nanoparticle and CNT electrode, the bR protein pump the protons $\left(\mathrm{H}^{+}\right)$to the silver nanoparticle catalyst following light absorption, promoting the adsorption and formation of $\mathrm{Ag}-\mathrm{H}$ adsorption on silver nanoparticle surfaces, which accelerates the rate determining step of the electrochemical hydrogen adsorption. Our bio-nano electrode has a metallic behavior due to Fermi levels within the conduction bands and improved conductivities with a delocalized nature of the active sites incorporated with silver nanoparticles on bR protein and thus increase the higher hydrogen evolving reaction activity. Thus we speculate that the synergic effect of bR protein, $\mathrm{Ag}$ NP and CNT's are important for enhancing reduction of protons to hydrogen. 


\section{Experimental}

\section{Printing}

The stereolithographic printer employed for printing the bionano ink is Ember 3D printer by Autodesk equipped with light engine using a high-power $405 \mathrm{~nm}$ LED with DLP digital micro-mirror device (DMD) containing over 1 million mirrors spaced 7.6 microns apart in a compact footprint.

\section{Bio nano ink composition}

Acrylic monomers, di(ethylene glycol)dimethacrylate (dEGdMA) with $300 \mathrm{ppm}$ monomethyl ether hydroquinone as inhibitor and 2-hydroxyethyl methacrylate (2-HEMA) with $250 \mathrm{ppm}$ monomethyl ether hydroquinone as inhibitor and tetra acrylic cross linker, di(trimethylolpropane)tetraacrylate (dTMP4A) with 1000 ppm monomethyl ether hydroquinone as inhibitor were purchased from Sigma-Aldrich. The inhibitor was removed by passing through a basic alumina column and degassed by passing nitrogen prior to bio-nano ink preparation. Photo initiator diphenyl(2,4,6-trimethylbenzoyl)phosphine oxide/2hydroxy-2-methylpropiophenone-blend was bought from Aldrich. bR protein was recombinantly expressed and purified using molecular biology techniques. Cross linkable $\beta$-sheet peptide was designed and synthesized in lab using AAPPTec Infinity 2400 and lyophilized over 2 days (Labconco FreeZone 4.5 plus). All amino acids and preloaded resins were purchased from AAPPTec LLC. (Louisville, KY, USA) and used as received. The $\beta$-sheets are assembled in the outer core of bR stabilizing the membrane protein. ${ }^{26}$ Silver nano particle were prepared using standard protocol and single walled CNT was purchased from NanoIntegris. The optimization of the composition was done by trial and error method, firstly subjecting to UV lamp

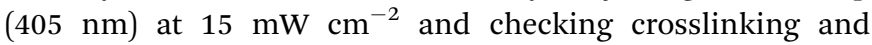
secondly fine-tuning the composition by slice by slice printing with stereolithographic printer.

\section{Characterization}

Developed bio-nano ink and printed structures were analyzed for following traits.

3(a) Material characterization. The $\beta$-sheet peptides were purified using reverse-phase high-performance liquid chromatography (HPLC) (AAPPTec UV3000 \& two P3000) working with a Spirit Peptide $120 \mathrm{C} 1810 \mu \mathrm{m} 25 \times 2.12$ column (AAPPTec) and analyzed with a liquid chromatography/mass selective detector (LC/MSD) (Agilent 1100 Series) using a Zorbax SB-C18 $5 \mu \mathrm{m} 4.6$ $\times 250 \mathrm{~mm}$ (Agilent) analytical column and mass spectrometer set to positive-ion mode. The stability of the wrapped protein was assessed using circular dichroism (CD), Jasco J-810 spectropolarimeter. The sustainability of the proton pump was confirmed using UV-Visible Spectroscopy, PerkinElmer-Lambda 1050. Silver nano particles were characterized using Transmission Electron Microscope (Hitachi HF 3300 TEM). Varian FTS 7000 FT-IR Imaging System measured the composition of printed bio nano inks.

3(b) Thermal analysis. TA instruments, TGA Q50 is used for thermo gravimetric analysis (TGA) and DSC Q2000 is used for differential scanning calorimetry (DSC) studies. The TGA samples weighed $9.0 \pm 0.5 \mathrm{mg}$, ranging in temperature from ambient to $600{ }^{\circ} \mathrm{C}$. DSC samples weighed 4.0-5.0 mg, with a temperature cycle ranging from $-50-300{ }^{\circ} \mathrm{C}$. All experiments were conducted in dynamic nitrogen atmosphere, flow rate $10 \mathrm{~mL} \mathrm{~min}{ }^{-1}$, heating rate $10{ }^{\circ} \mathrm{C} \mathrm{min}^{-1}$, alumina crucible for TG, and tzero hermetic pan and lid for DSC.

3(c) Rheology. Discovery HR-3 hybrid rheometer (TA instruments) is used for the rheology measurement of printed samples. $1 \mathrm{~mm}, 0.5 \mathrm{~mm}$ and $0.3 \mathrm{~mm}$ thick and $2.5 \mathrm{~mm}$ diameter samples were printed and characterized via testing on a rheometer (DHR-3, TA Instruments) with a $25 \mathrm{~mm}$ diameter, Peltier plate steel geometry. Strain sweep experiments were conducted with logarithmic sweep of strain (0.0125-12.5\%) via fixed frequency of $1 \mathrm{~Hz}$. Oscillation experiments were conducted with a fixed oscillatory strain of $0.032 \%$, with a sweep of frequency $(0.015-16 \mathrm{~Hz})$. All experiments were in ambient conditions with a gap height in relation with sample thickness and preliminary soak time of $60 \mathrm{~s}$.

3(d) Surface morphology. Hitachi S-3000N Elevated Pressure Scanning Electron Microscope, and Hitachi S 4800 field emission scanning electron microscopy (FE-SEM), analyzed the element mapping and surface morphology of the printed materials respectively. Specific designs of $2 \mathrm{~mm} \times 1 \mathrm{~mm} \times$ $1 \mathrm{~mm}$ rectangular blocks with spicks $(1 \mathrm{~mm}$ long) and holes (1 mm deep) varying in diameter from $1 \mathrm{~mm}$ to $0.1 \mathrm{~mm}$ were developed using software Mesh and 3D printed and analyzed to quantify the resolution attainable.

3(e) Hydrogen evolution studies. The electrochemical experiments were carried out using Gamry electrochemical instrument having three-electrode with $\mathrm{Ag} / \mathrm{AgCl}$ reference electrode, $4 \mathrm{D}$ printed hydrogel as working electrode and a platinum wire as a counter electrode. The operating conditions were chosen based on a series of cyclic voltammetry measurements. The hydrogel was placed in a quartz cell and irritated with $535 \mathrm{~nm}$ light (intensity $50 \mathrm{~mW} \mathrm{~cm}^{-2}$ ) to activate the bR protein. The hydrogen generated was collected into a tube, and were collected from the cell using a gas-tight syringe and finally analyzed by gas chromatography. The hydrogen evolution was quantified by gas chromatography (Agilent Technologies 6890N Network GC system). Cyclic voltammetry (CV) experiments were tested in a standard three-electrode system on a Gamry Advance 3000 electrochemical workstation at room temperature to evaluate the device performance.

\section{Conclusions}

Present work focus on a deviation from the mainstream 4D printing of shape memory or mini tissue building and offers a versatile way of including bio traits and retaining functionality demonstrated by the successful stereolithographic printing of a bio photo electrochemical cell. The printing technique does not involve the extrusion process since the biomolecules are not strained during printing. The inherent function of $\mathrm{bR}$ as a proton pump is regenerated inside the printed structure and together with the combined effect of silver nanoparticles and carbon nanotubes the whole system can act as a bio photo 
electrochemical cell. The bio-nano ink designed relies on a combination of materials, stability and geometry that can be controlled inside an engineered space. The printed materials consist of pertinent thermo-mechanical properties that make them well suited for a wide range of applications. The efficiency of the photo electrochemical cell is found to be highly dependent on the thickness of the printed structure owing to the fact that thicker curing hinders the flexibility of the matrix as well as water uptake in between the polymer chains, straining the membrane protein bR out of its natural environment thereby restricting its conformational changes, enabling it, to act as proton pump. Our study enlightens a new angle of 4D printing by effectively stabilizing bio moiety in a pseudo membrane assembly together with nano tubes and particles equipping them to consort towards a specific function that provides features for designing of other novel bio-nano inks with improved bioactivity and durability, which leads to a plethora of applications.

\section{Conflicts of interest}

There is no conflicts to declare.

\section{Acknowledgements}

The authors thank Alberta Innovates Technology Futures (AITF) iCORE chair funding, National Institute of Nano Technology (NINT) Facilities and Antonio Jiménez for synthesizing peptide, Matthew Christensen for preparing $b R$ and Sumalee Salahub for FE-SEM operations.

\section{References}

1 C. Barnatt, 3D Printing - The Next Industrial Revolution, Chapter 1, 2013, http://www.ExplainingTheFuture.com/ 3Dprinting.

2 A. S. Gladman, E. A. Matsumoto, R. G. Nuzzo, L. Mahadevan and J. A. Lewis, Nat. Mater., 2016, 15, 413.

3 Y. Liu, J. K. Boyles, J. Genzer and M. D. Dickey, Soft Matter, 2012, 8, 1764.

4 J. A. Lewis, Adv. Funct. Mater., 2006, 16, 2193.

5 Q. Ge, A. H. Sakhaei, H. Lee, C. K. Dunn, N. X. Fang and M. L. Dunn, Sci. Rep., 2016, 6, DOI: 10.1038/srep31110.

6 Q. Ge, H. J. Qi and M. L. Dunn, Appl. Phys. Lett., 2013, 103, 131901.

7 D. Ratna and J. Karger-Kocsis, J. Mater. Sci., 2008, 43, 254.
8 Q. Zhao, W. Zou, Y. Luo and T. Xie, Sci. Adv., 2016, 2(1), e1501297.

9 R. M. Erb, J. S. Sander, R. Grisch and A. E. R. Studart, Nat. Commun., 2012, 4, 1712.

10 N. W. Bartlett, et al., Science, 2015, 349, DOI: 10.1126/ science.aab0129.

11 S. Tibbits, Architect. Digest., 2014, 84, 116.

12 L. Ionov, Macromol. Chem. Phys., 2012, 214, 1178.

13 J. H. Na, A. A. Evans, J. Bae, M. C. Chiappelli, C. D. Santangelo, R. J. Lang, T. C. Hull and R. C. Hayward, Adv. Mater., 2015, 27, 79.

14 J. Ryu, M. D'Amato, X. Cui, K. N. Long, H. J. Qi and M. L. Dunn, Appl. Phys. Lett., 2012, 100, 161908.

15 S. Miao, W. Zhu, N. J. Castro, M. Nowicki, X. Zhou, H. Cui, J. P. Fisher and L. G. Zhang, Sci. Rep., 2016, 6, 27226, DOI: 10.1038/srep27226.

16 F. Pati, J. Jang, D.-H. Ha, S. W. Kim, J.-W. Rhie, J.-H. Shim, D.-H. Kim and D.-W. Cho, Nat. Commun., 2014, 5, 3935, DOI: $10.1038 /$ ncomms4935.

17 J. W. Jung, J.-S. Lee and D.-W. Cho, Sci. Rep., 2016, 6, 21685, DOI: $10.1038 /$ srep21685.

18 D. Sundaramurthi, S. Rauf and C. A. E. Hauser, Int. J. Bioprint., 2016, 2, 9.

19 F. P. W. Melchels, J. Feijen and D. W. Grijpma, Biomaterials, 2010, 31, 6121.

20 D. W. Hutmacher, M. Sittinger and M. V. Risbud, Trends Biotechnol., 2004, 22, 354.

21 A. Fujishima and K. Honda, Nature, 1972, 238, 37.

22 Y. Jin, T. Honig, I. Ron, N. Friedman, M. Sheves and D. Cahen, Chem. Soc. Rev., 2008, 37, 2422.

23 L.-K. Chu, C.-W. Yen and M. A. El-Sayed, Biosens. Bioelectron., 2010, 26, 620.

24 N. K. Allam, C. W. Yen, R. D. Near and M. A. El-Sayed, Energy Environ. Sci., 2011, 4, 2909.

25 Z. Zhao, P. Wang, X. Xu, M. Sheves and Y. Jin, J. Am. Chem. Soc., 2015, 137, 2840.

26 H. Tao, et al., Nat. Methods, 2013, 10, 759.

27 C. Paul and J. P. Rosenbusch, EMBO J., 1985, 4, 1593.

28 C. E. Schafmeister, L. J. W. Miercke and R. M. Stroud, Science, 1993, 262, 734.

29 D. J. Gordon, K. L. Sciarretta and S. C. Meredith, Biochemistry, 2001, 40, 8237.

30 A. Kongkanand, R. M. Domínguez and P. V. Kamat, Nano Lett., 2007, 7, 676.

31 Y. Chun-Wan, S. C. Hayden, E. C. Dreaden, P. Szymanski and M. A. El-Sayed, Nano Lett., 2011, 11, 3821. 